\title{
Effect of amorphous interface layers on crystalline thin-film x-ray diffraction
}

\author{
T. Weisemoeller, F. Bertram, S. Gevers, C. Deiter, A. Greuling, and J. Wollschläger* \\ Fachbereich Physik, Universität Osnabrück, Barbarastrasse 7, D-49069 Osnabrück, Germany \\ and Center of Interface Science, Barbarastrasse 7, D-49069 Osnabrück, Germany
}

(Received 20 March 2009; published 19 June 2009)

\begin{abstract}
In this work, an analysis method of x-ray diffraction data of crystalline structures with amorphous interface layers is presented and applied to single crystalline films on amorphous interface layers. Thickness and morphology of crystalline films are obtained from x-ray diffraction at conditions where no significant interference effects between crystalline film and substrate occur. Extending the x-ray diffraction analysis to conditions where interference effects between the crystalline film and the substrate appear, it is also possible to determine the morphology of the amorphous interface film. The analysis method presented in this work is useful for the current and future analyses of amorphous structures between crystalline structures in general and is therefore applicable to many different material systems. This incorporates crystalline thin and ultrathin films on crystalline substrates as well as crystalline multilayers on crystalline and also on amorphous substrates. We apply the method developed here to characterize both crystalline praseodymia films and amorphous interface layers, which could be detected neither by $\mathrm{x}$-ray reflection nor by $\mathrm{x}$-ray diffraction previously.
\end{abstract}

DOI: 10.1103/PhysRevB.79.245422 PACS number(s): 61.05.cc, 61.05.cp, 68.55.-a, 77.55.+f

\section{INTRODUCTION}

$\mathrm{X}$-ray scattering techniques have been used in physics and materials science to gather information about structure and composition of materials and thin films. ${ }^{1}$ For instance, in $\mathrm{X}$-ray reflectivity (XRR), the intensity of a reflected beam is measured at small angles. ${ }^{2}$ Since the intensity of the reflected beam depends on both the angle of reflection and on the electron-density distribution of the analyzed material, information for both crystalline and noncrystalline materials can be obtained. X-ray diffraction (XRD), in contrast, relies on scattering and interference effects of materials with a longrange order. That means that only crystalline materials can be investigated directly using this method. Due to the extremely weak interaction of $\mathrm{x}$ rays with materials, both techniques are treated as nondestructive methods which can provide indepth information on a quantitative scale. XRR and XRD are averaging methods; resolution and precision depend strongly on the used radiation source. XRD data analysis can be accomplished on the basis of the kinematical theory of diffraction because multiple scattering effects are negligible.

The recorded data are usually fitted by simulation tools. These tools calculate expected data for certain assumed structures and compare this calculated data to the measured data. The parameters of the simulated sample are varied by fitting algorithms, so that a parameter set is found that describes the sample sufficiently. It is often problematic to find such a parameter set because too many variables are unknown. In XRR, for example, problems may occur if not only film thicknesses but also electron densities of the layers implied in the model are unknown. This is especially true for amorphous layers, as their exact stoichiometry is often unknown or if crystalline layer exposes many defects as vacancies or interstitials. As amorphous layers, in contrast to crystalline layers, are also not directly investigated by XRD because they have no long-range order, the properties of these layers are often not determined sufficiently.

In the following, we will develop a theory to determine the thickness of amorphous interface (IF) layers from XRD if a crystalline film overgrows the amorphous film. The results will be applied to the system praseodymium oxide on $\mathrm{Si}(111)$, where an amorphous film between the crystalline oxide film and the crystalline substrate is built out during postdeposition annealing processes. In principle, our calculations have some similarities to the determination of sites and interface distances of adsorbed monolayers obtained by surface $\mathrm{x}$-ray diffraction. ${ }^{3,4}$

In this work, ultrathin praseodymium oxide films are deposited on $\mathrm{Si}(111)$ and postdeposition annealed to transform the epitaxially grown hexagonal layer to a layer of cubic structure. Depending on temperature, oxygen pressure, and duration of the annealing process the stoichiometric composition can range from $\mathrm{Pr}_{2} \mathrm{O}_{3}$ to $\mathrm{PrO}_{2} \cdot{ }^{5-7}$ Also, an amorphous interface layer is formed during the annealing process. ${ }^{8}$ It consists of two sublayers. One of those is $\mathrm{SiO}_{2}$ rich and adjacent to the $\mathrm{Si}$ substrate. The other part of the interface is $\mathrm{Pr}$ rich and located between the $\mathrm{SiO}_{2}$-rich part of the interface and the crystalline praseodymium oxide film. The amorphous interface plays an important role in the phasetransformation process ${ }^{7}$ and in the field of isomorphic oxideon-oxide epitaxy. 9,10 However, it is hard to investigate. Surface sensitive methods such as X-ray photon spectroscopy (XPS) do not penetrate the oxide film of typically $5 \mathrm{~nm}$ thickness, deep enough to provide sufficient information. Sputter XPS is also problematic because the film and probably also the interface are influenced by the sputter process. XRR is basically successful, but quite unprecise, because the sublayers are partly too rough and/or thin and because not only their thicknesses but also their stoichiometries are unknown. In particular, the $\mathrm{Si}$ substrate and the $\mathrm{SiO}_{2}$-rich interface layer are not distinguishable because their electron densities are too similar. In this work we will demonstrate that it is possible to gain information about the amorphous interface thickness via XRD although diffraction is only sensitive to nonamorphous crystalline materials. Our work is done on the example of praseodymium oxide on $\mathrm{Si}(111)$ but is in general portable to many other material systems. 


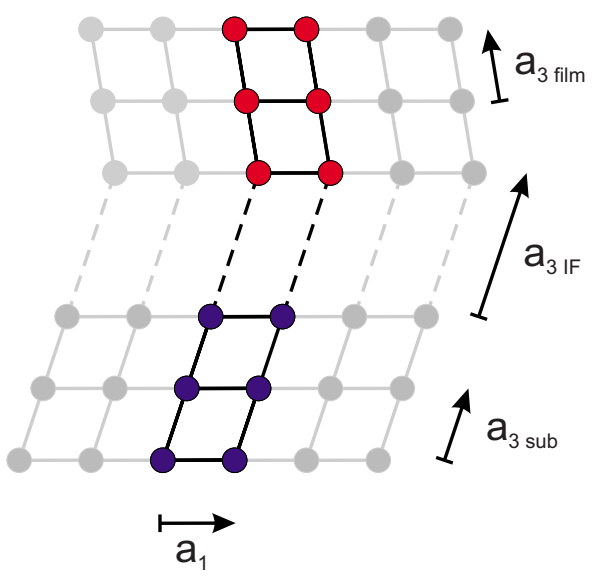

FIG. 1. (Color online) Exemplary model of two crystalline structures with variable distance between those structures. The unitcell boundaries of the substrate are represented by dark blue points; the ones of the crystalline film are represented by brighter red points. $\mathbf{a}_{2}$ (not shown here because the figure shows a 2D model) and $\mathbf{a}_{1}$ are parallel to the surface, whereas $\mathbf{a}_{3}$ has a component vertical to the surface.

\section{THEORY}

Diffraction of an incoming wave with the amplitude $A_{0}$ at a unit cell is described by the phase-correct summation of the waves diffracted at all atoms of the structure. This leads to the amplitude

$$
A(\mathbf{q})=A_{0} \cdot C \cdot F_{s}(\mathbf{q}) \cdot e^{i \cdot \mathbf{q} \cdot \mathbf{r}_{s}}
$$

of the diffracted wave with the scattering vector $\mathbf{q}=\mathbf{q}_{s}-\mathbf{q}_{i}$, where $\mathbf{q}_{i}$ is the wave vector of the incoming beam and $\mathbf{q}_{s}$ is the wave vector of the diffracted beam. The structural factor is defined as $F_{s}(\mathbf{q})$ and $\mathbf{r}_{s}$ is the position vector of the unit cell labeled $s .{ }^{4}$ The constant $C=\frac{e^{2}}{m c^{2}} \frac{1}{R_{0}}$ combines the influence of electron mass $m$, electron charge $e$, light velocity $c$, and distance between crystal and observer $R_{0}{ }^{1}$

A homogeneous structure with identical unit cells and identical structure factors $F_{s}(\mathbf{q})=F(\mathbf{q})$ that is infinite in two directions $(x$ and $y)$ and finite in one direction $(z)$, like a thin film, consists of an infinite number of the structures shown above,

$$
\begin{aligned}
A(\mathbf{q})= & A_{0} \cdot C \cdot \frac{1}{M_{x y n}} \cdot F(\mathbf{q}) \\
& \times \sum_{x=-\infty}^{\infty} \sum_{y=-\infty}^{\infty} \sum_{n=0}^{N-1} \exp \left[i \cdot \mathbf{q}\left(x \mathbf{a}_{1}+y \mathbf{a}_{2}+n \mathbf{a}_{3}\right)\right] .
\end{aligned}
$$

$\mathbf{a}_{i}$ are the unit-cell vectors, $N$ is the number of layers, and $M_{x y n}$ is the normalization constant, which is equal to the number of unit cells. $\mathbf{a}_{1}$ and $\mathbf{a}_{2}$ are unit vectors in the surface plane, while the unit vector $\mathbf{a}_{3}$ has a component vertical to the surface plane (cf. Fig. 1). Due to interference, for most scattering vectors $\mathbf{q}$, this amplitude equals zero because of the two infinite sums. Only if $\mathbf{q} \cdot \mathbf{a}_{1}=2 \pi H$ and $\mathbf{q} \cdot \mathbf{a}_{2}=2 \pi K$ with $H, K \in \mathbb{Z}$, the function does not equal to zero [twodimensional (2D) Laue conditions]. In other words, this is the case when the Bragg condition in $H$ and $K$ directions is fulfilled. If this is the case, Eq. (2) is reduced to

$$
A\left(H, K, \mathbf{q} \cdot \mathbf{a}_{3}\right)=A_{0} \cdot C \cdot \frac{1}{M_{n}} \cdot F(\mathbf{q}) \cdot \sum_{n=0}^{N-1} \exp i \cdot \mathbf{q} \cdot \mathbf{a}_{3} \cdot n,
$$

which is the scattering amplitude of a single column. In the following, we simulate the intensity of the diffracted wave only on crystal truncation rods [CTRs $(H, K \in \mathbb{Z})]$ and can therefore describe the crystallographic structure sufficiently as a one-dimensional stacking of unit cells as described in Eq. (3) (column model). An exemplary model is shown in Fig. 1. In order to define a three-dimensional (3D) reciprocal space with $(H K L)_{S}$ coordinates with respect to the surface of the Si substrate, we expand our 2D Laue conditions to threedimensional conditions (infinite 3D crystal) by $\mathbf{q} \cdot \mathbf{a}_{3}=2 \pi L$. For the specular rod $\left(\mathbf{q}=\mathbf{q}_{\perp}\right)$, Bragg conditions for the $\mathrm{Si}$ substrate are fulfilled at $L \in \mathbb{Z}$.

Equation (3) describes systems with only one crystalline structure in general. We now choose a system of two crystalline structures to exemplary explain the interference effects we want to discuss in this work. From here on, we name the structures $s u b$ for substrate (semi-infinite crystal) and film for a film of a finite thickness of $N$ layers on this substrate because this is the material system which we will discuss in more detail in this work. However, it is important to keep in mind that in principle these interference effects occur between any crystalline structures. Our model is valid if the lateral unit vectors for substrate and films $\mathbf{a}_{1}$ and $\mathbf{a}_{2}$ are identical. Thus the film has to be pseudomorphic. The third unit vector $\mathbf{a}_{3}$ with perpendicular component, however, may differ for substrate and film. Therefore, these vectors will be denoted $\mathbf{a}_{\text {sub }}$ and $\mathbf{a}_{\text {film }}$, respectively.

We choose a vector $\mathbf{r}_{\mathrm{IF}}$ to describe the distance between the two interfering crystal structures. For $N=1$, this exactly describes the well-known surface diffraction from monolayers and submonolayers with superstructure, where $\left|\mathbf{r}_{\mathrm{IF}}\right|$ is an atomic distance. Here, however, we will especially consider the effect of amorphous interface layers which do not directly contribute to the diffraction signal due to the missing long-range order of amorphous material. Therefore, $\left|\mathbf{r}_{\mathrm{IF}}\right|$ can be much larger than $\left|\mathbf{a}_{\text {sub }}\right|$ and $\left|\mathbf{a}_{\text {film }}\right|$. The amplitude of the wave diffracted at the double structure characterized above is thus described as

$$
A(\mathbf{q})=A_{\mathrm{sub}}(\mathbf{q})+A_{\mathrm{film}}(\mathbf{q}) \cdot e^{i \cdot \mathbf{q} \cdot \mathbf{r}_{\mathrm{IF}}} .
$$

For the simulations shown in this work, a column of unit cells is used as shown in Fig. 1. $\mathbf{a}_{\text {sub }}$ is the lattice vector between two adjacent unit cells of the substrate and $\mathbf{a}_{\text {film }}$ is accordingly the lattice vector between unit cells of the film. $\mathbf{r}_{\mathrm{IF}}$ is the vector pointing from the topmost unit cell of the substrate to the lowest unit cell of the film. The amplitude of a wave diffracted at the substrate in this column model is 


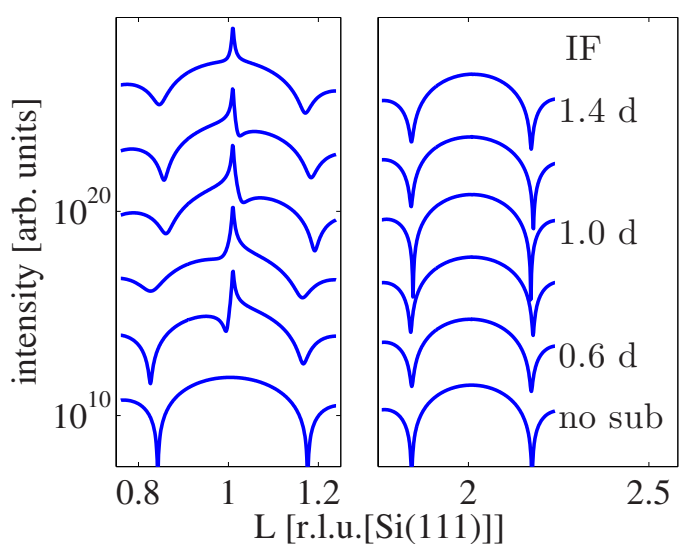

FIG. 2. (Color online) Simulation of diffracted intensity vs scattering vector of six layers $\mathrm{PrO}_{2}(111)$ on a $\mathrm{Si}(111)$ substrate for $H$ $=K=0$. The distance between film and substrate is influenced by the interface layer thickness IF and is varied from $0.6 d$ to $1.4 d$, where $d$ is the vertical lattice constant of the $\mathrm{PrO}_{2}(111)$ surface unit cell. For comparison, a simulation of the oxide layer alone is also included. It is clearly recognizable that interference effects occur between substrate and oxide at $L \approx 1$ and that the interface has a considerable effect on these interference effects, whereas the interference effects are negligible for $L \approx 2$.

$$
\begin{aligned}
A_{\mathrm{sub}}(\mathbf{q}) & =\sum_{n=-\infty}^{0} F_{\mathrm{sub}}(\mathbf{q}) \cdot \exp \left[\left(i \cdot \mathbf{q} \cdot \mathbf{a}_{\mathrm{sub}}+\epsilon(\mathbf{q})\right) \cdot n\right] \\
& =\frac{F_{\mathrm{sub}}(\mathbf{q})}{1-e^{-\epsilon(\mathbf{q})} e^{-i \cdot \mathbf{q} \cdot \mathbf{a}_{\mathrm{sub}}}}
\end{aligned}
$$

Here we introduced the damping of the amplitude $\boldsymbol{\epsilon}(\mathbf{q})$ due to absorption of $\mathrm{x}$-ray intensity which relies on the electron density of the substrate and on the incidence angle, as well as on the scattering angle. The latter is defined by the scattering vector $\mathbf{q}$ at a given wavelength of the diffracted wave, while the angle of incidence is obtained for $\mathbf{q}_{i}$ with respect to the surface.

The amplitude of the wave diffracted at a thin film of $N$ layers is calculated as

$$
A_{\mathrm{film}}(\mathbf{q})=\sum_{n=0}^{N-1} F_{\mathrm{film}}(\mathbf{q}) \cdot e^{i \cdot \mathbf{q} \cdot \mathbf{f}_{\mathrm{film}} \cdot n}=F_{\mathrm{film}}(\mathbf{q}) \frac{1-e^{-i \cdot \mathbf{q} \cdot \mathbf{a}_{\mathrm{film}} N}}{1-e^{-i \cdot \mathbf{q} \cdot \mathbf{a}_{\mathrm{film}}}} .
$$

Due to the small finite thickness of the film, damping effects are neglected here.

\section{SIMULATED DATA}

Figure 2 shows the simulated intensity of a specular $\theta-2 \theta$ $\mathrm{XRD}$ scan for a thin praseodymium oxide film with a thickness of six layers on a $\mathrm{Si}(111)$ substrate. The intensity of the diffracted beam is plotted versus the scattering vector. Labeling of the axes corresponds to the hexagonal $\mathrm{Si}(111)$ lattice in surface coordinates $(H K L)_{S}$. In this case lateral distances are irrelevant $(H=K=0)$ as we scan the specular $(00 L)_{S}$ rod. In order to keep things simple, we chose the $\mathrm{PrO}_{2}$ unit cell, which consists of only three atoms, to simulate the oxide layer. Again for reasons of simplicity, we assume that the oxide film is grown pseudomorphic so that lattice constants of substrate and oxide are the same both in real and reciprocal space. Figure 2 shows broad praseodymium oxide peaks at $L=1$ and $L=2$, whereas a sharp Si peak appears only at $L=1$. The Si peak at $L=2$ is symmetry forbidden in the kinematical theory of diffraction due to the diamond structure of $\mathrm{Si}$. Thus the right part of the figure, which shows the simulated data around $L=2$, is not affected by the Si substrate significantly. The simulation was made for five different IF thicknesses between substrate and oxide. An IF thickness of $1.0 \mathrm{~d}$ denotes the distance between substrate and oxide of exactly one layer distance of $\operatorname{PrO}_{2}(111)$, which means $3.135 \AA$.

At $L \approx 2$, the intensity distributions of the diffracted wave are in good approximation identical for different interface layer thicknesses. This demonstrates that the influence of the interface layer thickness is negligible here. At $L \approx 1$, in contrast, the intensity distribution depends clearly on the interface layer thickness.

From Eq. (4), we can calculate the intensity of the diffracted wave as

$$
I=\left|A_{\text {sub }}\right|^{2}+\left|A_{\text {film }}\right|^{2}+A_{\text {sub }}^{*} \cdot A_{\text {film }} \cdot e^{i \mathbf{q} \mathbf{r}_{\mathrm{IF}}}+A_{\text {sub }} \cdot A_{\text {film }}^{*} \cdot e^{-i \mathbf{q} \mathbf{r}_{\mathrm{IF}}} .
$$

This shows that the thickness of the interface layer only plays a role for scattering vectors under which the amplitudes of the waves diffracted at film and substrate are on the same order of magnitude. If $A_{\text {sub }} \gg A_{\text {film }}$, Eq. (7) can be approximated to $I=\left|A_{\text {sub }}\right|^{2}$. If $A_{\text {sub }} \ll A_{\text {film}}$, it can be approximated to $I=\left|A_{\text {film }}\right|^{2}$. As the Si Bragg peak on the specular rod at $L=2\left[\mathrm{Si}(002)_{S}\right.$ in surface coordinates and $\mathrm{Si}(222)_{B}$ in bulk coordinates] is symmetry forbidden, only negligible interference effects can be observed here, whereas around the $\mathrm{Si}(001)_{S}$ Bragg peak, interference effects cause the asymmetrical intensity distribution of the diffracted wave. The intensity slightly left (and right) of the Si Bragg peak is most dependent on the interface layer thickness. At $L \approx 0.98$, for example, the simulated intensity shows a local minimum for an interface thickness of $0.6 d$ due to destructive interference between the waves diffracted at the substrate and at the film, whereas a much higher intensity due to constructive interference appears at the same position for an interface thickness of $1.2 d$.

The influence of substantially bigger interface layers $\left(r_{\mathrm{IF} \perp} \gg a_{\mathrm{film} \perp}, a_{\mathrm{sub} \perp}\right)$ is demonstrated in Fig. 3. Here, a wider interval of the specular rod in the reciprocal space is presented compared to Fig. 2. Apart from this and the bigger interface layers, the simulated systems of the two figures are identical. One can see that the intensity distributions of the scattered waves are different for different interface layer thicknesses and that these differences occur not only at $L$ $\approx 1$ but also at lower and slightly higher scattering vectors. On the other hand, the intensity at $L \approx 2$ is not affected significantly by varying the interface distance. It shows almost exactly the intensity of the nonsupported film (no substrate). We already discussed that these different intensity distributions are due to interference effects between substrate and 


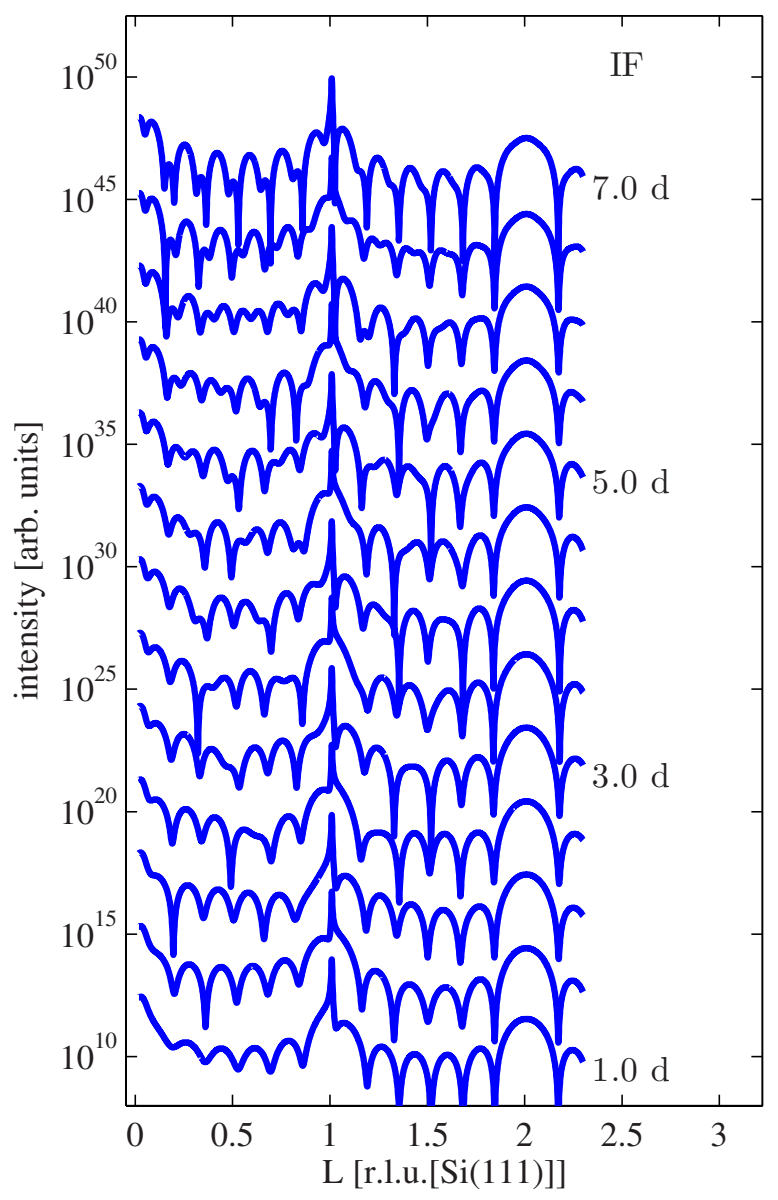

FIG. 3. (Color online) Simulation of the diffracted intensity vs scattering vector of six layers $\mathrm{PrO}_{2}(111)$ on a $\mathrm{Si}(111)$ substrate for $H=K=0$. The distance between substrate and oxide is varied significantly from $1.0 \mathrm{~d}$ to $7.0 \mathrm{~d}$. Interference effects cause additional intensity oscillations except for the region around the kinematically forbidden $\mathrm{Si}(002)_{S}$ peak, where the $\mathrm{Si}$ signal is too weak to cause noticeable interference effects.

oxide and that these do only appear if the diffracted amplitudes at film and substrate are in the same order of magnitude. Again, this is the reason that almost no interference effects are observed at $L \approx 2$ because the amplitude of the wave diffracted at the Si substrate is very low here due to the forbidden $\operatorname{Si}(002)_{S}$ Bragg reflex. For the model with a six layer thick oxide film $(N=6)$, we observe what we call double oscillations at $L \approx 1$ for an interface layer thickness of also six layer distances. This behavior can easily be understood by taking a look at the interference part of Eq. (7). The amplitude of the film oscillates with $e^{i \cdot 6 \cdot \mathbf{q} \mathbf{a}_{\text {film }}}[\mathrm{cf}$. Eq. (6) $]$ and the interference part also oscillates with an additional $e^{ \pm \mathbf{i q r} \mathbf{r}_{\mathrm{IF}}}$ with $\mathbf{q r}_{\mathrm{IF}}=6 \cdot \mathbf{q} \mathbf{a}_{\text {film }}$. If this oscillation interferes with the nonvanishing but nonoscillating diffracted amplitude $A_{\text {sub }}$ from the substrate, the result is an increased number of oscillations, which is no longer proportional to the thickness of the film but proportional to the added up thickness of film and interface layer. This, of course, is only visible if the amplitudes of film and oxide are in the same order of magnitude. This is the case close to the Si Bragg condition $(L \approx 1)$ and not close to the symmetry forbidden Si Bragg condition ( $L$ $\approx 2$ ).

\section{EXAMPLE OF APPLICATION: PRASEODYMIUM OXIDE ON Si(111)}

An ultrathin praseodymium oxide layer on $\mathrm{Si}(111)$ is a rather complicated system for quantitative $\mathrm{x}$-ray diffraction and $\mathrm{x}$-ray reflection analysis. The reason is that praseodymium oxide has many different stoichiometries that are hard to distinguish because of their similar crystalline structures and unit-cell sizes. This problem is increased as phases with different stoichiometries tend to coexist in powder praseodymia as well as in ultrathin films. ${ }^{7,11}$

An ultrathin hexagonal $\mathrm{Pr}_{2} \mathrm{O}_{3}$ film was deposited on a clean $\mathrm{Si}(111)$ wafer and postdeposition annealed at $700{ }^{\circ} \mathrm{C}$ in 1 atm oxygen to transform the film into a cubic $F m \overline{3} m$ $\mathrm{PrO}_{2}$ structure following a recipe that was reported in more detail previously. ${ }^{6,7}$ Thereafter, the sample was annealed in UHV at $300{ }^{\circ} \mathrm{C}$ to increase the interface thickness. It was shown that after the UHV annealing process the oxide film was reduced to cubic $\operatorname{Pr}_{2} \mathrm{O}_{3} .{ }^{12}$ In order to keep things simple, we chose the $\mathrm{PrO}_{2}$ unit cell, which consists of only three atoms, to simulate the oxide layer. The difference to other praseodymia unit cells, e.g., $\operatorname{Pr}_{2} \mathrm{O}_{3}$, is negligible for the simulations shown in this work. ${ }^{13}$

Investigation of the silicate interface layer, which was formed between substrate and film via XRR is a rather difficult process because the exact stoichiometry of the oxide film and of the interface layer between film and substrate is not known. It was shown before that this kind of interface layers consists of two sublayers, namely, a Pr-rich one close to the oxide and an $\mathrm{SiO}_{2}$-rich one close to the substrate. ${ }^{7,8}$ The thickness of the latter is extremely hard to determine via $\mathrm{XRR}$, as its electron density and, consequently, its index of refraction is close to the one of the $\mathrm{Si}$ substrates. Here we will show how to overcome these problems by XRD analysis of the interference effects between film and substrate. Experimental data presented in this work were collected at beamline W1/HASYLAB.

In Ref. 12, the sample was analyzed using a two column model to accommodate the lateral coexistence of two stoichiometric phases of the oxide film. In order to keep things simple, we will stick with a simple one-column model here because the character of the interface turned out to be identical for both columns in Ref. 12. The first step of our XRD interface analysis is to reduce the amount of free parameters by incorporating XRD analysis results of the measurements at $L \approx 2$, where $n o$ significant interference between oxide and film occurs because the intensity of the symmetry forbidden $\mathrm{Si}(002)_{S}$ is very weak. We can determine oxide film thickness $(20$ layers of $3.26 \AA=65 \AA)$ and surface roughness $(4 \AA)$ with high accuracy from these data [cf. Fig. 4 (right)]. The one column model used here is sufficient to describe the data for our needs because the lattice constants of the two coexisting stoichiometries are very close to each other, as was also shown in Ref. 12. At $L \approx 1$, the analysis of the diffracted intensity is depending extremely strongly on marginal differences in the parameter set. This results in a num- 


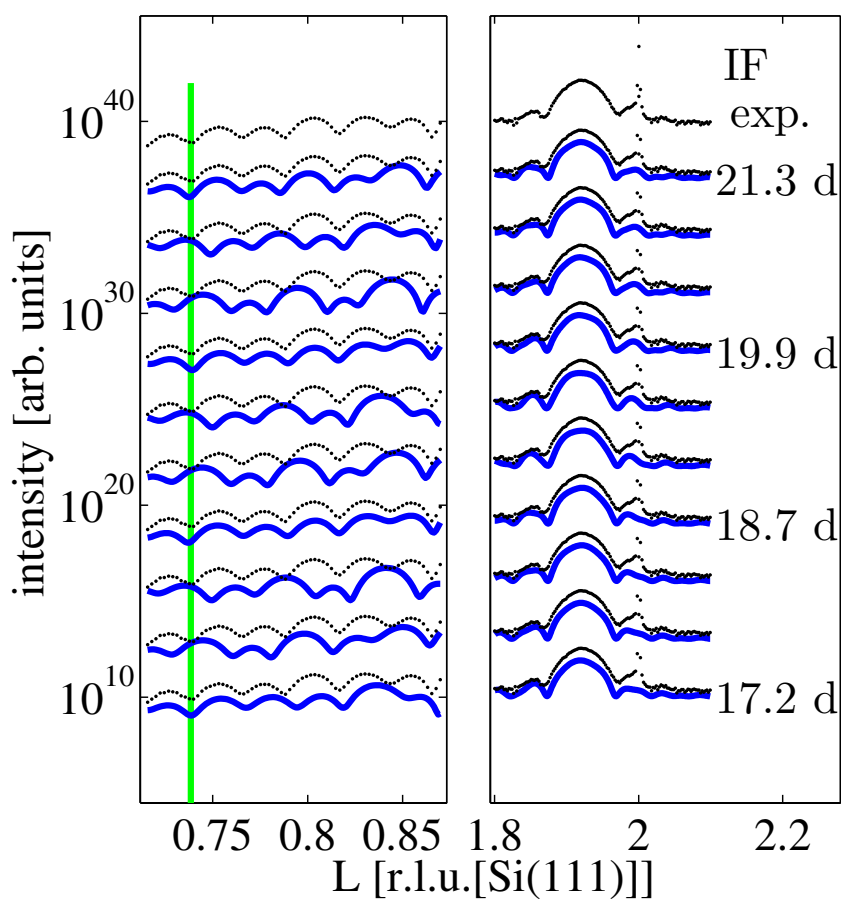

FIG. 4. (Color online) Simulation of the diffracted intensity of a postdeposition annealed praseodymium oxide on $\mathrm{Si}(111)$ sample (blue lines) and experimental data (black dots). The thickness of the amorphous interface layer IF, which causes interference effects, is varied from 17.2 to $21.3 \mathrm{PrO}_{2}(111)$ layer distances $d$. The $\mathrm{Si}$ signal at $L=2$ is very weak because the Bragg condition is symmetry forbidden. Therefore, interference effects in this region are also very weak.

ber of local minima in the goodness of fit (GOF) and is the reason why an XRD analysis of the $L \approx 1$ region has not been performed successfully for the given material system up to this point.

However, if one goes to slightly smaller scattering vectors than $L=1$, as shown in Fig. 4 (left), the analysis is possible. This is due to the fact that the coexisting stoichiometries have different surface roughnesses and hence only the phase with the lowest roughness shows fringes at scattering vectors that differ considerably from the Bragg peak positions. The blue lines in Fig. 4 show fitting curves for different interface layer thicknesses from $r_{\mathrm{IF} \perp}=17.2 d\left[17.2 \mathrm{PrO}_{2}(111)\right.$ layer distances $d=3.26 \AA$ each] to $r_{\mathrm{IF} \perp}=21.3 d$. All other parameters were kept constant for this figure. Measured data are plotted in black as comparison. The green line at $L=0.74$ marks a local intensity minimum of the measured data. The position of the local minimum depends on the position of the Bragg peak and the distance between the fringes around this peak. The latter depends on the film thickness as well as on the interface thickness as shown above, whereas the Bragg peak position depends on the vertical lattice constant alone. All parameters except for the interface layer thickness are well known from the analysis of the region at $L \approx 2$. That means that the positions of the local minima are characteristic of a certain interface layer thickness. As the peak positions shift when the interface layer thickness is increased, most interface layer thicknesses can be ruled out just by checking whether the simulated function shows a local mini-

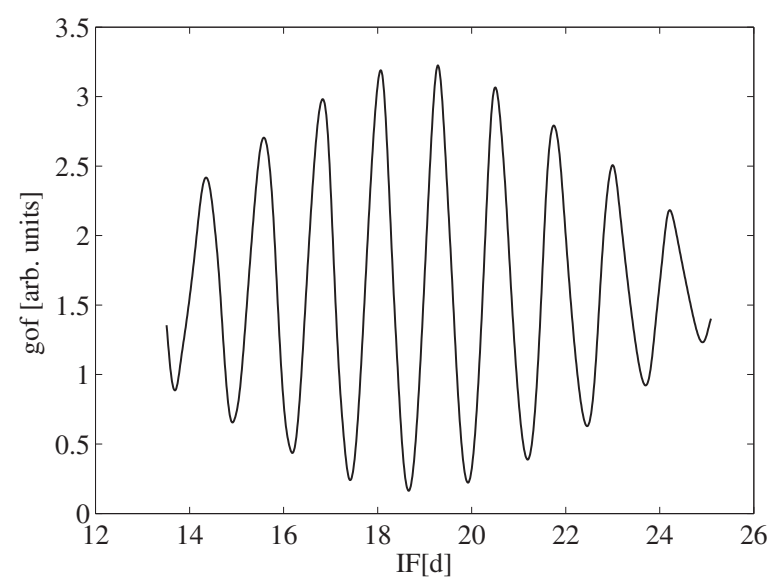

FIG. 5. GOF vs interface layer thickness in $\mathrm{PrO}_{2}(111)$ layer distances $d$ for the simulation also shown in Fig. 4. Only the region with noticeable interference effects $(0.715<L<0.870)$ is accounted for here. Lower GOF values stand for a better fitting function. Although only one parameter is varied, the GOF cycles many local minima and maxima.

mum at $L=0.74$ or not. This is only the case for interface layer thicknesses of 17.2, 18.7, 19.9, and $21.3 \mathrm{PrO}_{2}$ layer distances $d$. If we include the positions of other local minima, especially of the one at $L=0.87$, it is obvious that the distance between local minima is too short if the interface layer thickness is set to above $19.9 d$ and that the distance is too long if the interface layer thickness is below $18.7 d$.

In Fig. 5, the GOF is plotted versus the interface layer thickness in more detail. The GOF oscillates strongly with increasing interface layer thicknesses. These oscillations are due to the shifting of the intensity minima in reciprocal space that was also discussed above. The positions of the simulated minima mainly match the positions of the measured intensity minima when the GOF is in a local minimum, whereas the positions of the simulated maxima match the positions of the measured intensity maxima when the GOF is in a local maximum. These maxima and minima of the GOF are most pronounced if the distance between the minima in the simulated curve matches the distance of the minima in the measured curve most accurately.

In summary, we can determine the thickness of the amorphous interface layer with very high accuracy as 18.7 $\times 3.26 \AA=61 \AA$ or $19.9 \times 3.26 \AA=65 \AA$. This is regardless of the difficult material system of at least two coexisting stoichiometries of the thin praseodymia film on the Si substrate. In Ref. 12, the thickness of the amorphous interface layer was calculated as only $49 \AA$ from XRR experiments (cf. Fig. 6). This interface layer thickness, however, is only the thickness of the Pr-rich silicate part since XRR is not sensitive to the $\mathrm{SiO}_{2}$-rich part. Therefore, the difference is due to the close electron densities of the $\mathrm{SiO}_{2}$-rich part of the interface layer and the $\mathrm{Si}$ substrate, which makes the $\mathrm{SiO}_{2}$ part of the interface layer undetectable via XRR. The difference between the interface layer thicknesses measured via $\mathrm{XRR}$ and XRD is thus equal to the thickness of the $\mathrm{SiO}_{2}$-rich part of the interface layer, which is thus $14 \pm 2 \AA$. This is in good agreement with the thickness obtained by Ono and 


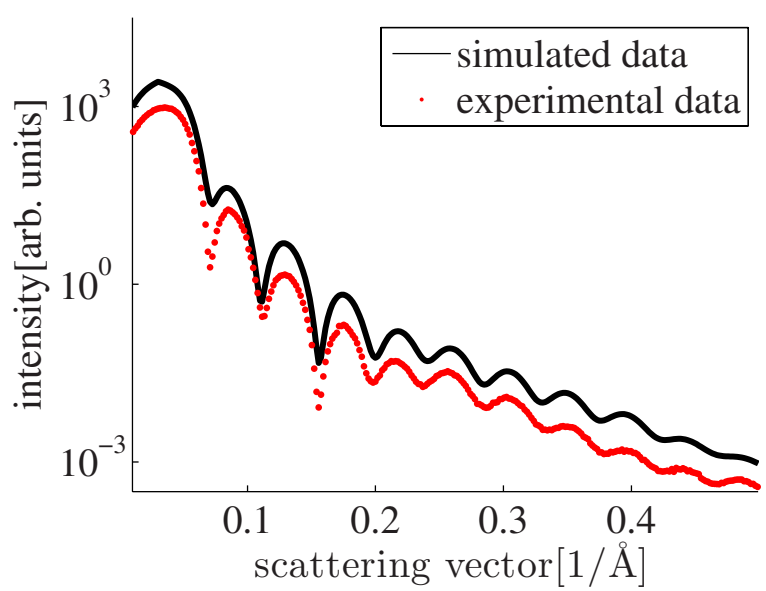

FIG. 6. (Color online) XRR simulation and experimental data from Ref. 12 of the praseodymium oxide on Si(111) sample discussed in this work. The thickness of the amorphous interface layer is determined as only $49 \AA$ via XRR in contrast to the thickness of $63 \pm 2 \AA$ calculated from XRD data in this work. The difference is due to the $\mathrm{SiO}_{2}$-rich part of the interface layer, which is not detectable via XRR.

Katsumata $^{8}$ via Fourier transform infrared. Ono and Katsumata found out that the interface layer for different rareearth oxides (including praseodymium) on Si consists of two sublayers as introduced above. The thickness of the $\mathrm{SiO}_{2}$-rich part of the interface layer is independent of the rare-earth element used and was estimated to be between 15 and $25 \AA$ for samples annealed in $600{ }^{\circ} \mathrm{C}$ oxygen at $1 \mathrm{~atm} .{ }^{8}$ Gevers et al..$^{12}$ found out that the thickness of the interface layer increases when $\mathrm{PrO}_{2}$ samples are annealed in UHV and thus reduced to $\mathrm{Pr}_{2} \mathrm{O}_{3}$. Combining our findings with the ones of Gevers et al. and Ono and Katsumata, we can conclude that the increase is due to an increase in the Pr-rich part of the interface and not in the $\mathrm{SiO}_{2}$-rich part.

\section{SUMMARY}

The presented XRD method is suitable for the analysis of $\mathrm{x}$-ray diffraction interference effects between crystalline structures in general. It is best applicable for systems where XRD results both with and without interference effects are obtainable. In the case presented here, these are results on the specular rod at $L=1$ and $L=2$. However, on less complicated material systems, without coexisting phases of different stoichiometries with similar crystalline structures, this method should be applicable even if regions without interference effects do not occur. The method presented here is especially promising for grazing incidence x-ray diffraction (GIXRD) on nonspecular rods. As shown above, the influence of interference effects depends on the relative intensities of the waves diffracted at the specific structures (in this case the praseodymium oxide film structure and the $\mathrm{Si}$ substrate structure). In GIXRD, these relative intensities depend on the (variable) incidence angle of the $\mathrm{x}$-ray beam and can thus be adjusted as required to a certain degree. In addition, selecting different diffraction rods permits the analysis of diffraction with and without interference effects for a wide range of material systems.

\section{ACKNOWLEDGMENTS}

XRD experiments were carried out at the synchrotron light source facilities DORIS III at HASYLAB/DESY. DESY is a member of the Helmholtz Association (HGF). We would like to thank Wolfgang Calibe for assistance in using beamline W1 and Wolfgang Drube for assistance in using beamline BW2. We also like to thank Thomas Schroeder and Alessandro Giussani from Innovations for High Performance Microelectronics (IHP) for providing the sample used in this work. *joachim.wollschlaeger@uos.de

${ }^{1} \mathrm{~J}$. Als-Nielsen and D. McMorrow, Elements of Modern X-ray Physics (Wiley, New York, 1999).

${ }^{2}$ M. Tolan, X-Ray Scattering from Soft-Matter Thin Films (Springer, New York, 1999).

${ }^{3}$ R. Feidenhansl, Surf. Sci. Rep. 10, 105 (1989).

${ }^{4}$ I. K. Robinson and D. J. Tweet, Rep. Prog. Phys. 55, 599 (1992).

${ }^{5}$ T. Schroeder, P. Zaumseil, G. Weidner, Ch. Wenger, J. Dabrowski, H.-J. Müssig, and P. Storck, J. Appl. Phys. 99, 014101 (2006).

${ }^{6}$ T. Weisemoeller, C. Deiter, F. Bertram, S. Gevers, A. Giussani, P. Zaumseil, T. Schroeder, and J. Wollschläger, Appl. Phys. Lett. 93, 032905 (2008).

${ }^{7}$ T. Weisemoeller, F. Bertram, S. Gevers, A. Greuling, C. Deiter,
H. Tobergte, M. Neumann, A. Giussani, T. Schroeder, J. Wollschläger, J. Appl. Phys. (to be published).

${ }^{8}$ H. Ono and T. Katsumata, Appl. Phys. Lett. 78, 1832 (2001).

${ }^{9}$ T. Schroeder, I. Costina, P. Storck, A. Wilke, O. Seifarth, A. Giussani, H.-J. Mussig, and P. Zaumseil, J. Appl. Phys. 103, 084102 (2008).

${ }^{10}$ A. Schaefer, T. Schroeder, G. Lupina, Y. Borchert, J. Dabrowski, Ch. Wenger, and M. Bäumer, Surf. Sci. 601, 1473 (2007).

${ }^{11}$ V. S. Rudenko and A. G. Boganov, Inorg. Mater. 7, 98 (1971).

${ }^{12} \mathrm{~S}$. Gevers, T. Weisemoeller, B. Zimmermann, F. Bertram, C. Deiter, J. Wollschläger, J. Phys.: Condens. Matter 21, 175408 (2009).

${ }^{13}$ A. Greuling, M.S. thesis, University of Osnabrück, 2007. 